\title{
In-situ Measurement of Crystalline Lattice Strains in Polytetrafluoroethylene
}

\author{
E.N. Brown • P.J. Rae • D.M. Dattelbaum • B. Clausen • \\ D.W. Brown
}

Received: 24 January 2007 / Accepted: 16 July 2007 / Published online: 11 August 2007

(C) Society for Experimental Mechanics 2007

\begin{abstract}
Strain measurements by neutron diffraction are employed as an in situ technique to obtain insight into the deformation modes of crystalline domains in a deformed semi-crystalline polymer. The SMARTS (Spectrometer for MAterials Research at Temperature and Stress) diffractometer has been used to measure the crystalline lattice displacements in polytetrafluoroethylene (PTFE) for crystalline phase IV (at room temperature) in tension and compression and for crystalline phase I (at $60^{\circ} \mathrm{C}$ ) in compression. The chemical structure of PTFE, $-\left(\mathrm{C}_{2} \mathrm{~F}_{4}\right)_{-}$, makes it ideally suited for investigation by neutron methods as it is free of hydrogen that results in limited penetration depths and poor diffraction acquisition in most polymers. Deformation parallel to the prismatic plane normals is shown to occur by inter-polymer chain compression with a modulus $\sim 10 \times$ bulk, while deformation parallel to the basal plane normal occurs by intra-polymer chain compression with a modulus $\sim 1000 \times$ bulk, corresponding with theoretical values for a PTFE chain modulus. Deformation parallel to the pyramidal plane normals is accommodated by interpolymer chain shear.
\end{abstract}

\author{
E.N. Brown ( $₫$, SEM member) · P.J. Rae · D.W. Brown \\ MST-8, Los Alamos National Laboratory, \\ MS G-755, \\ Los Alamos, NM 87545, USA \\ e-mail: en_brown@lanl.gov \\ D.M. Dattelbaum \\ DE-9, Los Alamos National Laboratory, \\ Los Alamos, NM 87545, USA \\ B. Clausen \\ LANSCE-LC, Los Alamos National Laboratory, \\ Los Alamos, NM 87545, USA
}

Keywords Polytetrafluoroethylene - PTFE - Lattice strain · Time-of-flight neutron diffraction

\section{Introduction}

While PTFE is known to be semi-crystalline in nature, the effect of mechanical deformation on the crystalline structure and comparative relationships to bulk behavior has received very limited attention. The bulk constitutive response of well pedigreed PTFE (DuPont Grade 7C) has recently been published for tension and compression $[1,2]$. In the current work, we extend these studies to specifically examine crystalline lattice displacements in PTFE 7C for crystalline phase IV (at room temperature) in tension and compression and for crystalline phase I $\left(\right.$ at $\left.60^{\circ} \mathrm{C}\right)$ in compression using the SMARTS (Spectrometer for Materials Research at Temperature and Stress) diffractometer at the Los Alamos Neutron Science CEnter (LANSCE). The chemical structure of PTFE, $-\left(\mathrm{C}_{2} \mathrm{~F}_{4}\right)_{-}$, makes it ideally suited for investigation by neutron methods as it lacks hydrogen in its chemical structure that limits penetration depths and results in poor diffraction measurements in most polymers. For example, the related polymer polyethylene is employed for neutron moderation at the LANSCE facility (for a discussion of the forms of polyethylene see [3]). Previous applications of SMARTS have been focused on metallic materials (see for example [4-9]). This is partially due to difficulties in interpreting diffraction patterns of amorphous materials [10], and partially because of the relatively few polymers that could be successfully probed in the bulk by diffraction methods. In the current work we extend the application of in situ strain measurement techniques to this semi-crystalline polymer, presenting results for diffraction peaks corresponding to basal, pris- 
matic, and pyramidal modes of deformation within the crystalline domains of PTFE 7C.

The phase behavior of PTFE, as first reported by Bunn and Howells [11], exhibits two atmospheric pressure crystalline transitions at $19^{\circ} \mathrm{C}[11]$ and $30^{\circ} \mathrm{C}[12]$ that reflect changes in both short and long range ordering of the polymer chains. The first-order transition at $19^{\circ} \mathrm{C}$ between phases II and IV represents an untwisting in the helical conformation from 13 atoms $/ 180$ degree turn $[12,13]$ to 15 atoms/turn $[12,14,15]$ [Fig. 1(a)] and an associated increase in the hexagonal lattice spacing [Fig. 1(b)]. Further rotational disordering and untwisting of the helices occurs above $30^{\circ} \mathrm{C}$ giving way to phase I to form a pseudo-hexagonal structure in which the individual polymer chains lose their well defined helical repeat unit $[12,16]$. The semi-crystalline nature of PTFE is illustrated in Fig. 1(c) in which isolated crystalline domains on the order of 5 to $20 \mu \mathrm{m}$ are connected by amorphous polymer (image obtained by tapping mode AFM [17]). Amorphous PTFE has the same repeat atomic structure as the crystalline domains but without significant order. The extreme molecular weight of PTFE $\left(\sim 1 \times 10^{6}\right)$, allows a given polymer chain to assemble in both amorphous and crystalline regions [18].

\section{Experimental}

\section{Sample Preparation}

The pedigreed PTFE polymer investigated in the current work was manufactured from PTFE 7C molding powder acquired from DuPont. Billets measuring $600 \times 600 \times 65 \mathrm{~mm}$ were pressed and sintered by Balfor Industries (NY) following ASTM D-4894-98a resulting in a crystallinity of approximately $38 \%$ (by DSC) [2]. Values of crystallinity and density for the pedigreed PTFE $7 \mathrm{C}$ are provided in Table 1. A general review of methods for measuring crystallinity in PTFE has been presented by Lehnert et al. [19] including a discussion of variability in results. The mechanical properties of the pedigreed PTFE 7C have been extensively characterized [1, 2, 17, 20-24]. These earlier works also contain extensive reviews of the literature. Samples were machined from the pressed and sintered billets of pedigreed PTFE 7C with the loading in the inplane directions while ensuring a nominal temperature rise to prevent changes in the material crystallinity.

For tension measurements an ASTM D638 Type I specimen is employed with a nominal thickness of $8 \mathrm{~mm}$. Data for the room temperature tensile response is presented out to a true strain of $15 \%$. For compression measurements right cylinders $20 \mathrm{~mm}$ tall by $10 \mathrm{~mm}$ diameter are used. Two sets of measurements are presented for the room temperature compressive response investigated to true strains of 60 and $5 \%$. This demonstrates both the far-field and lattice strain repeatability. The compressive response of PTFE at $60^{\circ} \mathrm{C}$ is investigated to a true strain of $19 \%$. Although PTFE has a room temperature true strain to failure of $\sim 160 \%$ [1], the sample size required in conjunction with the maximum crosshead displacement limit the applied strain to $15 \%$. However, diffraction only provides the Hookean response of the crystalline domains, so with the exception of the one compression test to a true strain of $60 \%$ the focus was on smaller strain measurements presented.

The SMARTS diffractometer is built around a horizontal custom-built Instron hydraulic load frame, which is used in the current work to load specimens in either tension or
Fig. 1 Phase IV crystalline PTFE exhibits both (a) a shortrange helical structure within the individual polymer chains and (b) long range hexagonal packing structure between chains. (c) PTFE is a semi-crystalline polymer with the crystalline domains separated by regions of amorphous PTFE
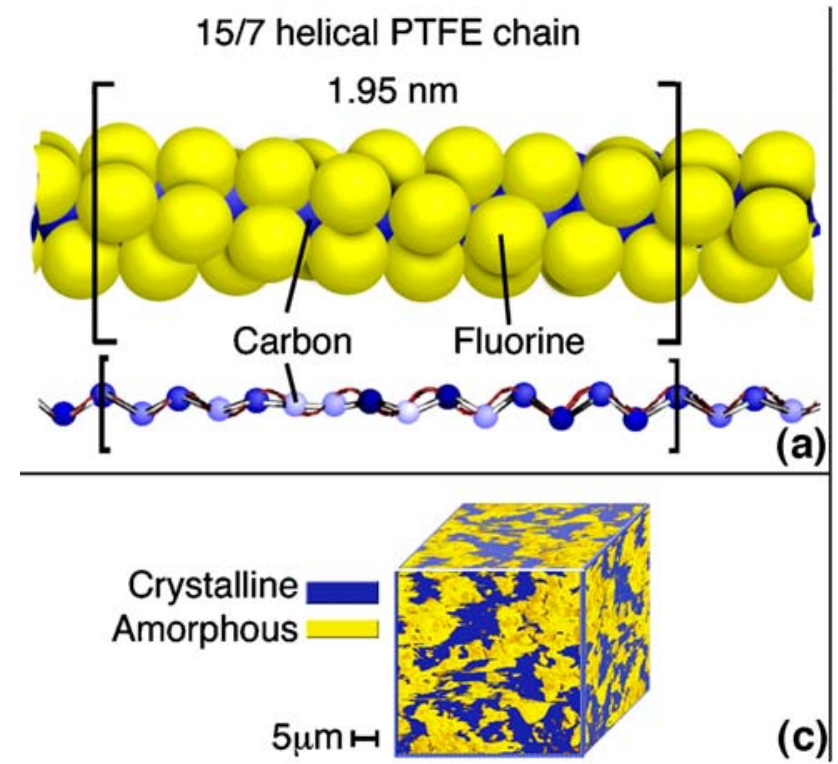

(c)

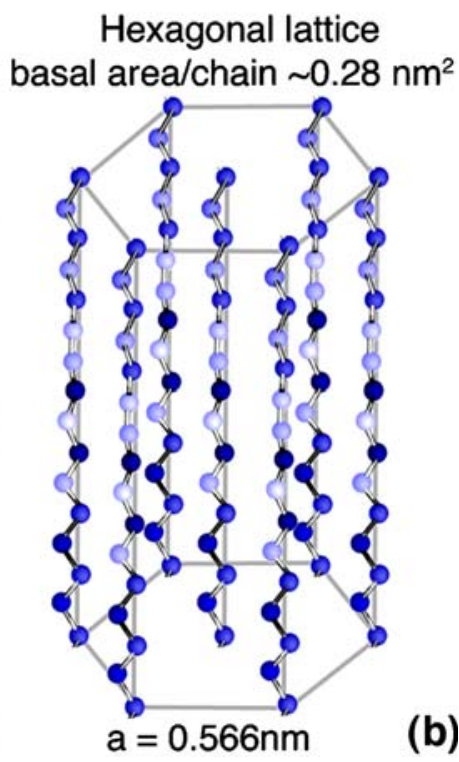


Table 1 Crysallinity values for PTFE 7C by standard methods [2]

\begin{tabular}{llllll}
\hline Density $\left(\mathrm{kg} / \mathrm{m}^{3}\right)$ & \multicolumn{5}{c}{ Crystallinity (\%) } \\
\hline He-pycnometry & Immersion & DSC & Density & IR & WAXS \\
$2168.9 \pm 0.1$ & $2169.6 \pm 0.1$ & $38 \pm 1$ & $53 \pm 1$ & $73 \pm 10(7 A)$ & $69 \pm 2$ \\
\hline
\end{tabular}

compression. Samples were loaded under constant engineering stain rate of $10^{-4} \mathrm{~s}^{-1}$ while measuring bulk stress-strain response (Fig. 2) with a $10 \mathrm{kN}$ load cell and knife-edge extensometer. Due to the horizontal configuration of SMARTS a small preload was necessary to prevent misalignment of the sample prior to testing of compression samples. The sample was held under constant cross-head conditions-yielding nominally constant strain-during diffractions measurements with an acquisition time of $\sim 1 \mathrm{hr}$. Substantial viscous relaxation was observed during these rest periods, which was experimentally unavoidable. Despite this, the outer stress-stain envelope resulting from the loading profile in the current work exhibits close correlation with previous data for this pedigree of PTFE 7C for a continuous constant strain rate test. This was true for all data sets obtained at LANSCE.

\section{Neutron Diffraction}

The lattice strain measurements were made on the SMARTS time-of-flight diffractometer $[25,26]$ at LANSCE. LANSCE is a pulsed neutron source, which produces neutrons with a range of energies generated through spallation reactions in a tungsten target moderated by a water at $10^{\circ} \mathrm{C}$ [Fig. 3(a)]. The energies represent neutron velocities (and wavelengths) and their detection involves measuring the neutron times-of-

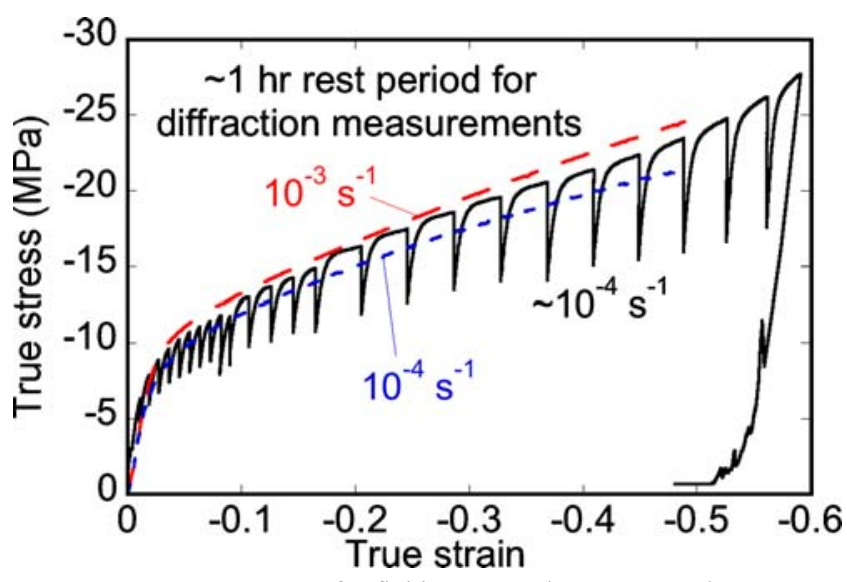

Fig. 2 Room temperature far-field compressive stress-strain response for continuous loading and with constant strain rest-periods for diffraction acquisition flight, at fixed angles from the sample. In terms of Bragg's law the diffraction angle $\theta$ is fixed while the wavelength $\lambda$ varies. This is the inverse of the more common method used with laboratory X-rays or reactor neutron sources, for which $\lambda$ is fixed and $\theta$ varies. Time-of-flight measurements are useful for lower symmetry structures, such as the hexagonal crystal lattices of crystalline PTFE domains. They enable unit cell parameters, and their changes, to be obtained from a wide range of diffraction peaks (from inter-planar or dspacings ranging typically from 0.5 to $4 \AA$ ). The sample was placed on the instrument XYZ $\theta$ stage and positioned optically with the aid of two Leica theodolites. The relationship for d-spacing vs. wavelength in the undeformed state for the diffractometer was calibrated with the aid of a standard $\mathrm{CaF}_{2}$ sample.

The instrument, illustrated in Fig. 3(b), has two detector banks consisting of $196{ }^{3} \mathrm{He}$ filled tubes, which are located $1.5 \mathrm{~m}$ from the sample situated at $\pm 90^{\circ}$ to the incident neutron beam and subtend roughly $20^{\circ}$ in the horizontal and vertical planes. The sample is mounted in the horizontal Instron load frame oriented at $45^{\circ}$ to the incident neutron beam. This enables a sample to be oriented such that diffraction data can be taken in the axial and transverse directions simultaneously. A schematic representation is shown in Fig. 3(c). Photographs of the inside of the instrument cave and the compression sample with mounted extensometer are shown in Fig. 3(d) and (e) respectively.

\section{Data Analysis}

The SMARTS diffractometer was designed to measure inter-planar or d-spacings of crystallographic lattices to a high degree of accuracy. The data were fitted with a convoluted Gaussian and Lorentzian peak shape on a peakby-peak basis with the Los Alamos GSAS code [27]. The lattice spacing, $d_{h k l}$, for each diffraction peak with Miller indices, $h k l$, was determined via Bragg's Law

$\lambda_{h k l}=2 d_{h k l} \sin \theta$,

where $\lambda_{h k l}$ is the wavelength associated with the $h k l$ reflection at a fixed angle of diffraction, $2 \theta$, equal to $90^{\circ}$.

The $d$-spacings (or changes therein) are then utilized as internal strain gages with strains calculated from the relation,

$\varepsilon_{h k l}=\left(d_{h k l}-d_{h k l}^{0}\right) / d_{h k l}^{0}+C\left(\varepsilon^{f f}\right)$,

where $d_{h k l}$ is the instantaneous $\mathrm{d}$-spacing and $d_{h k l}^{0}$ is measured for each reflection from a stress-free reference coupon. The high applied compressive strains, up to $60 \%$ true strain, used in the current work necessitate a correction $C$ to the lattice strain measurement accounting for the increase in cross sectional area of the specimen and 


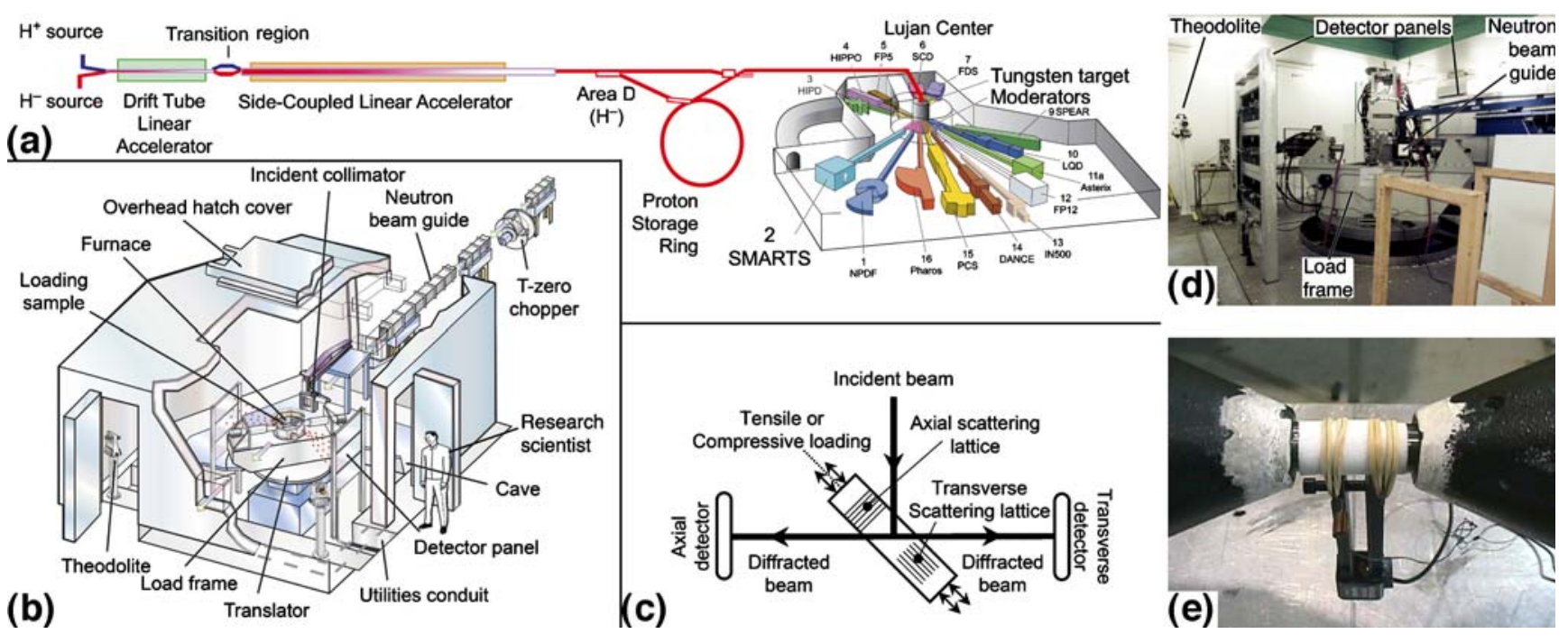

Fig. 3 Experimental set up. (a) The heart of the LANSCE user facility is a highly flexible linear accelerator (linac) system, one of the most powerful in the world, that can accelerate up to $1 \mathrm{~mA}$ of protons (from left to right) to an energy of $800 \mathrm{MeV}$ and then deliver the protons to multiple experimental areas. The accelerated negative hydrogen ions are injected into a $30 \mathrm{~m}$ diameter Proton Storage Ring (PSR). The PSR converts a $625 \mu$ s pulse of negative hydrogen ions into a $125 \mathrm{~ns}$ intense burst of protons. Those intense proton bursts are directed at the Lujan Center's tungsten target, which through nuclear spallation produces short bursts of neutrons. The SMARTS diffractometer (beam line \#2) used in the current research employs a water moderator to produce epithermal neutrons. Four views are presented for the detector and specimen layout of SMARTS: (b) a cutaway schematic of the experimental cave with a research scientist shown for scale, (c) a simplified schematic of the sample oriented at $45^{\circ}$ to the incident beam so that the diffraction vectors simultaneously measure the axial and transverse strains in the sample on the two detectors, (d) a photograph of the inside of the cave nominally looking along the neutron beam line, and (e) a close up of the compression sample with mounted extensometer. From the linac (measuring almost $1 \mathrm{~km}$ in length) to the measurement of microstrain in the crystalline lattice ( fm displacements) the experimental set up spans over 18 orders of magnitude in length. Between these extremes lie the load frame and experimental cave $(\sim \mathrm{m})$, the samples $(\sim 10 \mathrm{~mm})$, the crystalline domains $(\sim 10 \mu \mathrm{m})$, and the crystalline lattice dimensions $(\sim 0.1 \mathrm{~nm})$

movement of the center of mass from the calibration scenario. The corrections are given by

$C_{\text {Longitudinal }}=\left\{\begin{array}{ll}0 & , \varepsilon^{f f}<0.11 \\ 280-2509 \times \varepsilon^{f f} & , \varepsilon^{f f}>0.11\end{array}\right.$ and $C_{\text {Transverse }}=-2601 \times \varepsilon^{f f}$,

where $\mathcal{E}^{f f}$ is the far field applied true strain [28]. Peak assignments were made based on published X-ray data on undeformed PTFE powder, filaments and thin films [29-33].

\section{Results}

Figure 4(a) and (b) show representative neutron diffraction patterns with peak assignments for phases IV and I respectively. The peak locations and relative intensities are
Fig. 4 Representative diffraction pattern for unloaded PTFE 7C overlaid with peak assignments (a) in phase IV at room temperature and (b) in phase I at $60^{\circ} \mathrm{C}$
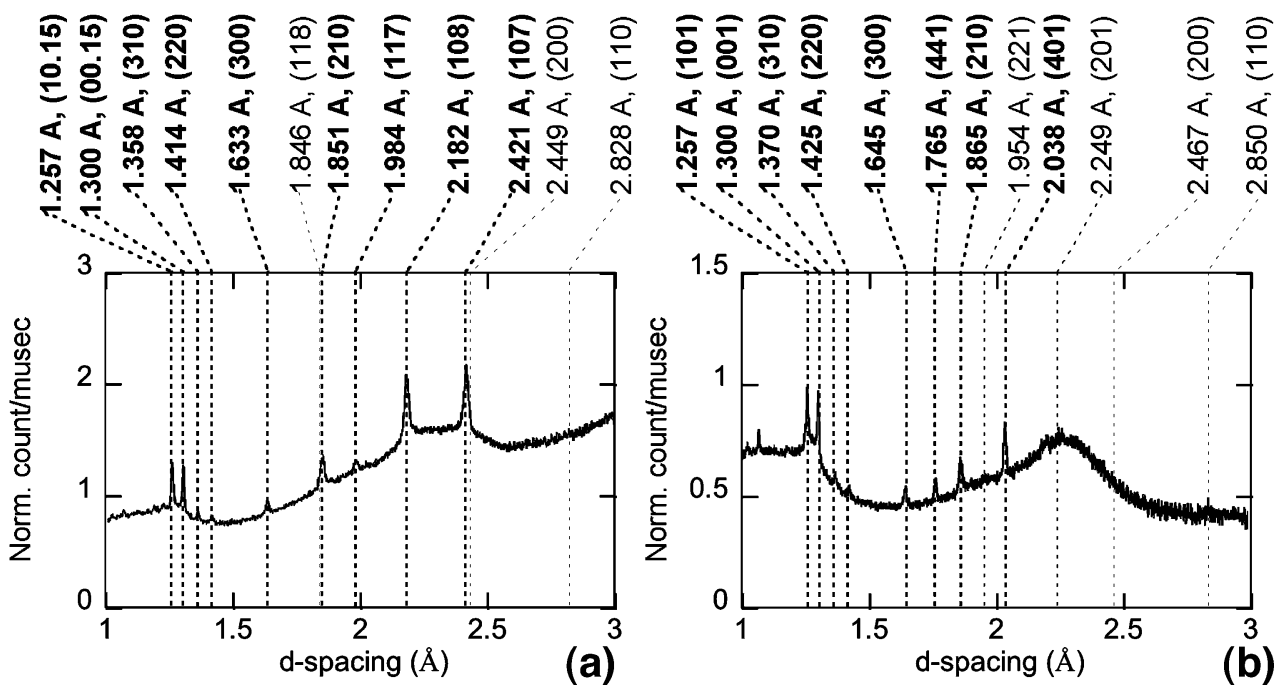
Table 2 Phase IV crystalline lattice constants

\begin{tabular}{|c|c|c|c|c|c|c|}
\hline \multirow{3}{*}{$\begin{array}{l}(\mathrm{hkl}) \\
100\end{array}$} & \multirow{3}{*}{$\frac{\text { d-space }(\AA)}{4.902}$} & \multirow{3}{*}{$\begin{array}{l}\text { Relative intensity } \\
\text { Off range }\end{array}$} & \multicolumn{4}{|c|}{ Intensity change $\left(I / I_{0}\right)$ at $10 \%$ strain } \\
\hline & & & \multicolumn{2}{|c|}{ Compression (axial/tran.) } & \multicolumn{2}{|c|}{ Tension (axial/trans.) } \\
\hline & & & na & na & na & na \\
\hline 110 & 2.822 & $\mathrm{~W}$ & na & na & na & na \\
\hline 200 & 2.423 & M (under 107) & na & na & na & na \\
\hline 107 & 2.424 & VS & $1.65 \pm 0.23$ & $0.80 \pm 0.04$ & $0.57 \pm 0.02$ & $0.40 \pm 0.07$ \\
\hline 108 & 2.178 & VS & $1.00 \pm 0.04$ & $0.74 \pm 0.03$ & $0.96 \pm 0.03$ & $0.46 \pm 0.02$ \\
\hline 117 & 1.986 & M & $2.37 \pm 0.25$ & $0.77 \pm 0.14$ & $0.67 \pm 0.05$ & $0.28 \pm 0.06$ \\
\hline 210 & 1.847 & $\mathrm{~S}$ & $4.61 \pm 0.15$ & $0.71 \pm 0.04$ & $0.94 \pm 0.05$ & $0.35 \pm 0.07$ \\
\hline 118 & 1.845 & M (under 210) & na & na & na & na \\
\hline 300 & 1.631 & M & $2.62 \pm 0.09$ & $0.77 \pm 0.07$ & $0.29 \pm 0.04$ & $0.91 \pm 0.07$ \\
\hline 220 & 1.411 & M & $2.06 \pm 0.11$ & $0.70 \pm 0.08$ & $0.33 \pm 0.05$ & $0.87 \pm 0.10$ \\
\hline 310 & 1.357 & M & $2.15 \pm 0.10$ & $0.66 \pm 0.07$ & $0.21 \pm 0.04$ & $0.76 \pm 0.08$ \\
\hline 00.15 & 1.299 & $\mathrm{~S}$ & $0.23 \pm 0.02$ & $1.14 \pm 0.02$ & $1.37 \pm 0.02$ & $0.40 \pm 0.01$ \\
\hline 10.15 & 1.257 & $\mathrm{~S}$ & $0.37 \pm 0.02$ & $1.09 \pm 0.02$ & $1.43 \pm 0.02$ & $0.38 \pm 0.01$ \\
\hline
\end{tabular}

Peak intensity is indicates as "VS" very strong, "S" strong, "M" medium, or "W" weak. Peaks that could not be well characterized because they were out of range, very weak or hidden by other peaks are denoted as "na."

given in Tables 2 and 3 for phases IV and I respectively. The measured neutron peaks correspond well with those reported from X-ray studies.

For phase IV the (100) plane with a d-spacing of $4.902 \AA$ is beyond the measurement range of SMARTS to measure, while the (110) peak is very weak compared to the background. The (200) and (118) peaks are obscured by the (107) and (210) peaks respectively. While slight asymmetry of the prominent peaks corroborate the presence of the masked peaks, deconvolution into the two peaks proved too noisy to capture the minor peak. The d-spacings are consistent with the unit cell shown in Fig. 1 (reported to be $a=5.66 \AA$ and $c=$ $19.5 \AA$ [29-33], calculated from current measurements to be $a=5.646 \pm 0.003 \AA$ and $c=19.485 \pm 0.000 \AA$ ). The lattice planes are illustrated in Fig. 5. The axial detector measures the orthogonal distance between these planes with the far field load applied normal to the planes; the transverse detector measures the orthogonal distance between these planes with the far field load applied parallel to the planes.

For phase I the (100) plane with a d-spacing of $4.935 \AA$ is beyond the range of SMARTS to measure and the (110), (200), (201), and (221) peaks are very weak compared to the background. The d-spacings are consistent with a unit cell of $a=5.70 \AA$ and $c=1.3 \AA$.

The far-field stress-strain response for the three test conditions are shown in Fig. 6. Unlike Fig. 2 where each

Table 3 Phase I crystalline lattice constants at $60^{\circ} \mathrm{C}$

\begin{tabular}{|c|c|c|c|c|}
\hline \multirow[t]{2}{*}{ (hkl) } & \multirow[t]{2}{*}{ D-space $(\AA)$} & \multirow[t]{2}{*}{ Relative intensity } & \multicolumn{2}{|c|}{ Intensity change $\left(I / I_{0}\right)$ at $10 \%$ strain } \\
\hline & & & \multicolumn{2}{|c|}{ Compression (axial/Transverse) } \\
\hline 100 & 4.935 & Off range & na & na \\
\hline 110 & 2.850 & $\mathrm{~W}$ & na & na \\
\hline 200 & 2.467 & $\mathrm{~W}$ & na & na \\
\hline 201 & 2.249 & $\mathrm{~W}$ & na & na \\
\hline 401 & 2.038 & M & $0.81 \pm 0.08$ & $0.90 \pm 0.03$ \\
\hline 221 & 1.954 & $\mathrm{~W}$ & na & na \\
\hline 210 & 1.865 & M & $1.31 \pm 0.03$ & $0.40 \pm 0.03$ \\
\hline 441 & 1.765 & M & $0.64 \pm 0.08$ & $0.84 \pm 0.04$ \\
\hline 300 & 1.645 & M & $1.26 \pm 0.04$ & $0.70 \pm 0.04$ \\
\hline 220 & 1.425 & M & $1.07 \pm 0.06$ & $0.67 \pm 0.10$ \\
\hline 310 & 1.370 & M & $0.92 \pm 0.06$ & $0.53 \pm 0.07$ \\
\hline 001 & 1.300 & $\mathrm{~S}$ & $0.23 \pm 0.07$ & $1.08 \pm 0.03$ \\
\hline 101 & 1.257 & S & $0.05 \pm 0.04$ & $1.04 \pm 0.04$ \\
\hline
\end{tabular}

Peak intensity is indicates as "VS" very strong, "S" strong, "M" medium, or "W" weak. Peaks that could not be well characterized because they were out of range, very weak or hidden by other peaks are denoted as "na". 
Fig. 5 Diffraction planes in phase IV PTFE

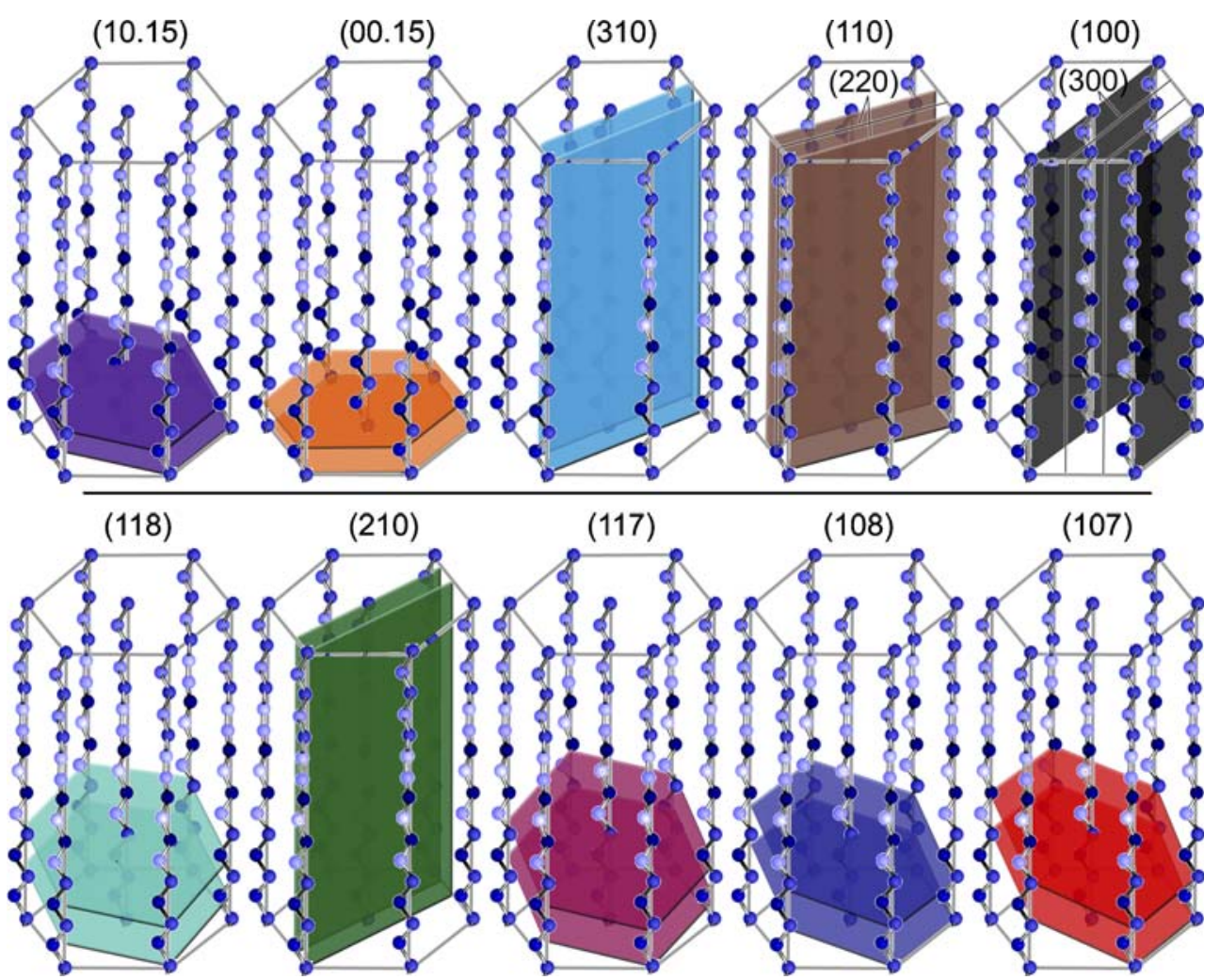

relaxation and reload is shown explicitly, for clarity only the bound of the loading profile during deformation are shown. The convention in the current work is that tensile stresses and strains are positive $(+)$ and compressive stresses and strains are negative $(-)$. With the exception of Fig. 6 where tension is in the first quadrant and compression is the third quadrant, all other plots have the increasing applied stress magnitude is positive along the y-axis. The corresponding strain direction is plotted along the positive $\mathrm{x}$-axis. According to this convention a classic axial material response appears the first quadrant, a classic Poisson

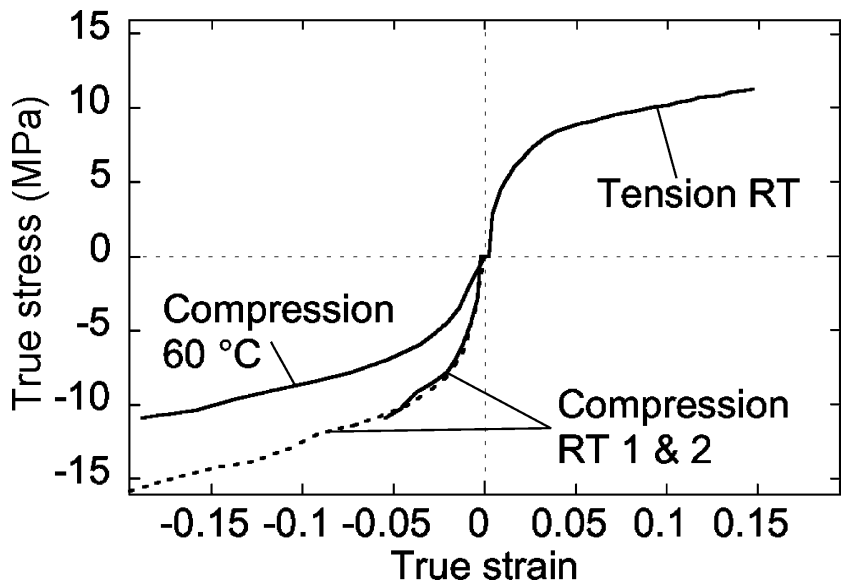

Fig. 6 Far-field stress-strain response for the four loading conditions measured. For clarity only the bound of the loading profile during deformation are presented response appears in the second quadrant. Figure 6 highlights the asymmetric behavior of PTFE with lower flow stress and strain hardening in tension. The compressive flow stress decreases significantly with an increase in temperature from room temperature to $60^{\circ} \mathrm{C}$.

Several diffraction peaks are observed to undergo significant changes in their shape in addition to the shift in peak location. Tables 2 and 3 report the ratio in intensitythe area between the Gaussian and Lorentzian peak shape and the local linearly fit background - between the measurements at $10 \%$ applied true strain $I$ and the unloaded conditions $I_{0}$. Under the three test conditions the most common response is a decrease in intensity, which can ultimately lead to peak extinction at higher strains. Notably, the maximum basal normal tensile strains-axial (00.15) and (10.15) under tension at room temperature, transverse (00.15) and (10.15) under compression at room temperature, and transverse (001) and (101) under compression at $60^{\circ} \mathrm{C}$-lead to increased intensity dominated by increased peak amplitude. Conversely, several of the axial responses under compressive loading exhibit increases in intensity due to peak broadening despite significantly reduced peak amplitude. Due to noise in the background these very short wide peaks can exhibit high error in intensity measurement and even greater error in the determining the center point $d$ spacing and subsequently calculating strain.

Figures 7, 8, and 9 show the diffraction lattice strains for the very strong through medium intensity peaks and the 
Fig. 7 Stress-strain response of the individual prismatic crystalline lattice planes for (a) phase IV in tension, (b) phase IV in compression, and (c) phase I in compression

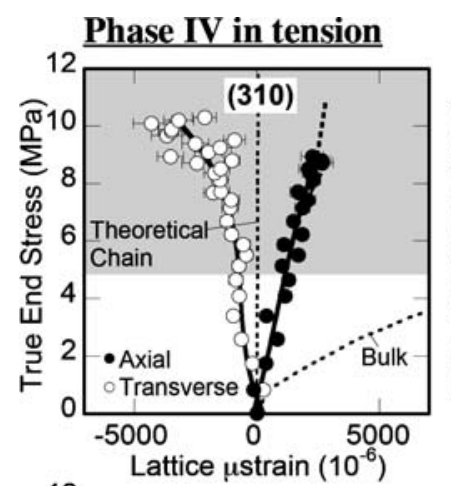

Phase IV in compression

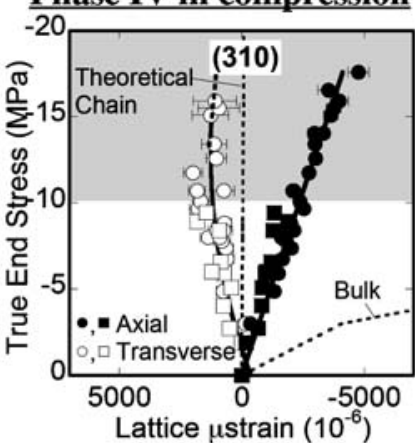

Phase I in compression
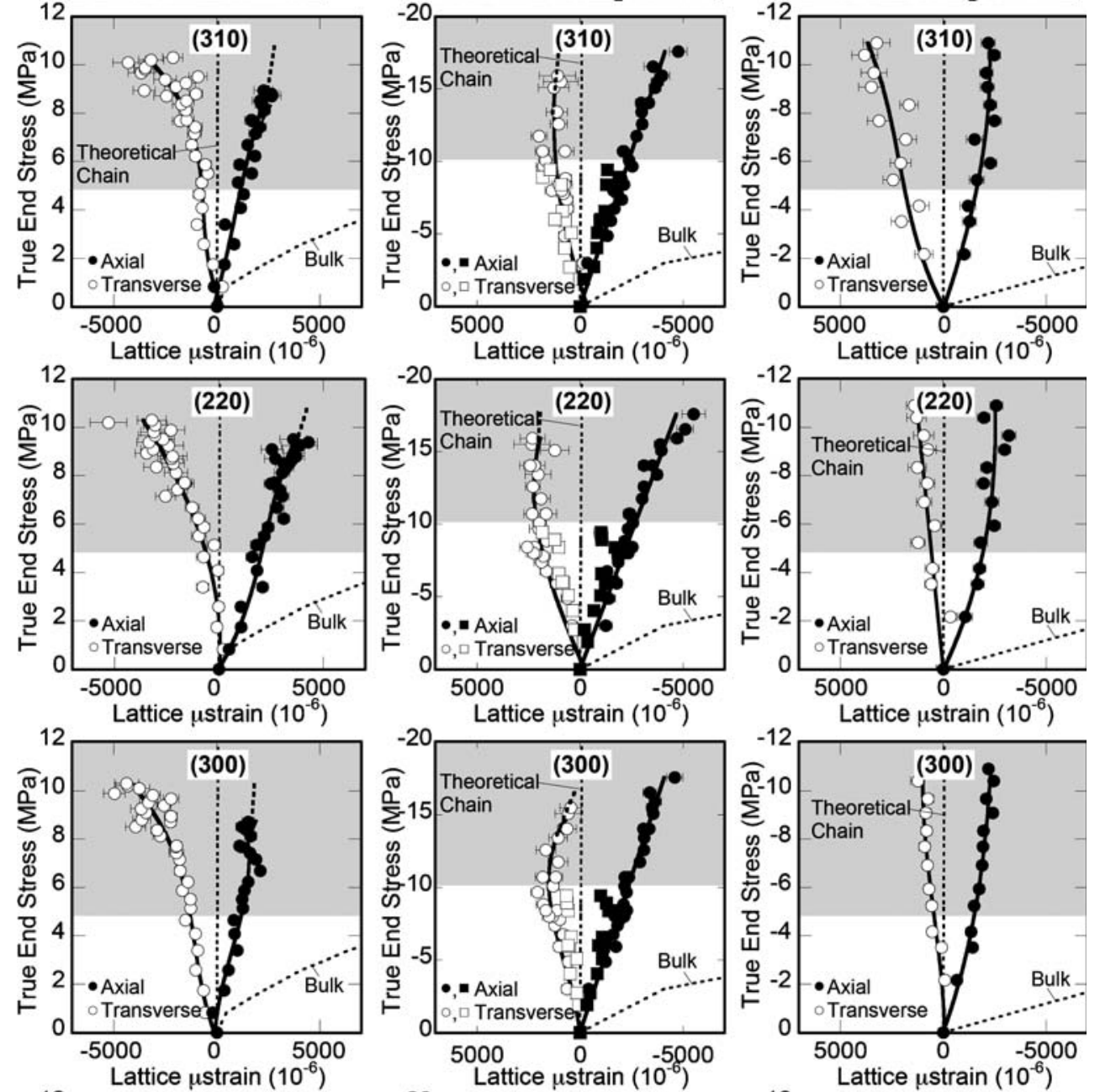

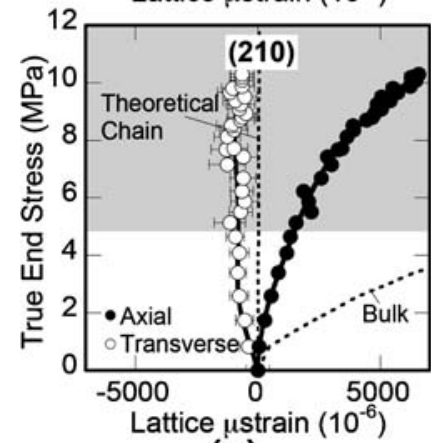

(a)

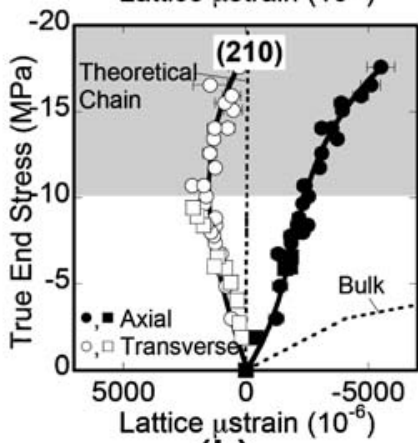

(b)

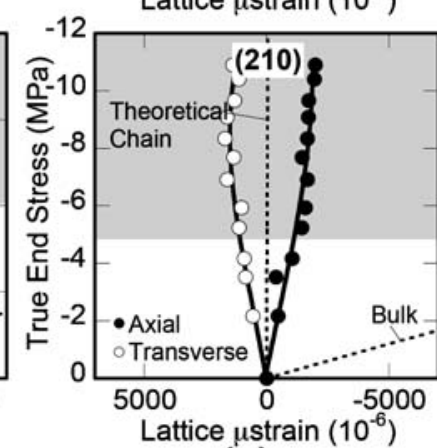

(c) three loading conditions, separated by prismatic, basal, and pyramidal orientations. They are plotted against the end relaxed far-field stress value corresponding to a given measurement. In each figure columns (a), (b), and (c) correspond to measurements for phases IV in tension, IV in compression, and I in compression respectively. The rows correspond to common crystalline lattices. For the prismatic crystalline lattice planes (Fig. 7) the same lattices are observed in both phases IV and I due do the common hexagonal packing of the polymer chains, albeit with slightly different $a$ values.
For the basal and pyramidal crystalline lattice planes (Figs. 8 and 9) different lattice planes arise due to the 15 atom helical repeat in phase IV versus the single atom random repeat along the polymer chain in phase I. In these cases similar lattice structures are shown in a given row. Each plot includes dashed traces indicating the measured bulk and theoretical single PTFE chain [34] stress-strain responses. The transition from a white to gray background indicates bulk yielding. The solid lines through the data are intended to serve as a guide-to-the-eye only, representing best-fit 
Fig. 8 Stress-strain response of the individual basal crystalline lattice planes for (a) phase IV in tension, (b) phase IV in compression, and (c) phase I in compression

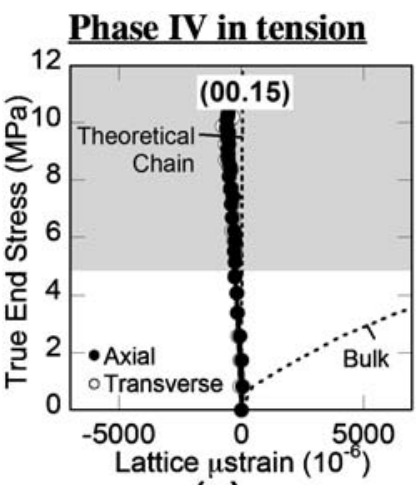

(a)
Phase IV in compression

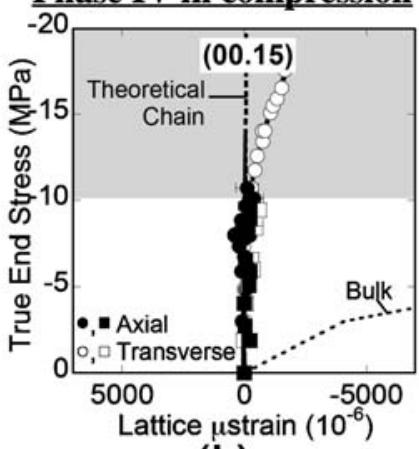

(b)

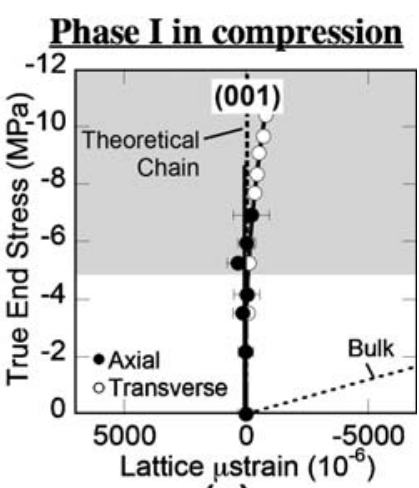

(c) polynomials of order between 0 and 4 . Although counter the classic bulk stress-strain yield response, a lattice response that tends towards the horizontal - such as the (210) crystal at room temperature-indicates not yielding but the taking on of extra load. Conversely, a lattice response that tends towards the veritcal - such as the prismatic crystal at $60^{\circ} \mathrm{C}$ indicates yielding based upon traditional polycrystalline diffraction measurements for metals where crystallographic deformation modes such as slip and twinning are the dominant deformation modes.

\section{Discussion}

Prismatic and Basal Plane Normal Behavior

In the elastic regime the crystalline lattice deforms in the way that would be expected on the basis of classic elasticity when loading is parallel to the prismatic plane normals: (310), (220), (300) or (210). The axial and transverse strains follow Hooke's law and Poisson's response respectively. The elastic response in the loading direction is bounded by the compliant bulk response of PTFE and stiff theoretical response of the PTFE polymer chain along the carbon backbone as reported by Bartha et al. [34] from a full-electron, crystal-orbital density functional method. In phase IV, with the exception of the (210) orientation, the axial response remains linear well after bulk plastic yielding has occurred. In Figs. 7, 8, and 9 a transition of the solid fitted curves to a dashed line indicates that either peak amplitude or intensity has diminished to the point that there is no longer confidence in the data.

In phase IV the crystalline domains with (310), (220), and (300) lattices the axial and transverse diffraction peaks disappear under tension and compression respectively. The two sets of compression measurements of phase IV have been overlaid consistently showing excellent agreement. In contrast to phase IV, in phase I the axial lattice strains saturate following bulk yielding. Strain saturation indicates that the imposed deformation is being accommodated by methods other than elasticity. In other words, in phase I, the prismatic grains axial lattice strains saturate following bulk yielding, deforming plastically or in-elastically. This may arise from the random helical progression of the polymer chains and larger hexagonal packing in phase I.

Under compression parallel to the basal plane normal(00.15) in phase IV and (001) in phase I-the axial response follows the theoretical polymer chain response [34], albeit with large error bars. The axial peaks disappear shortly after bulk yielding, likely due to orientation of these crystals out of the axial plane. Under far-field tensile loading the response of the (00.15) plane is less intuitive. While the axial (00.15) plane follows the theoretical chain response for the first three measurements with in the error bars, it deviates into a compressive strain well below bulk yielding. To further distinguish, the axial (00.15) diffraction peak increases under tension; the only peak in Phase IV to do so under either compressive or tensile loading.

There are also a few peaks that are observed in the diffraction pattern only under applied load. These peaks appear concomitant with the decrease of the initial peak intensities (as shown in Table 2). Since $d_{h k l}^{0}$ is unknown for these peaks, their strains cannot be calculated and they are not discussed in the current work. However, they are consistent with PTFE Phase III and this strain-induced phase transition is discussed in detail in a separate publication [35].

In polycrystalline metal the sum of the intensities of all of the peaks should remain constant and therefore the reduction of one peak must be compensated by the increase in another peak, whether it is due to texture development or phase changes. This is not the case for semicrystalline polymers because the volume of crystalline material, i.e., percent crystallinity, changes during deformation. The crystallinity of PTFE 7C has previously been reported to decrease under quasistatic deformation by DSC measurements [36]. While subjective, the finding from those DSC measurements may support the current qualitative decease 
Fig. 9 Stress-strain response of the individual pyramidal crystalline lattice planes for (a) phase IV in tension, (b) phase IV in compression, and (c) phase I in compression
Phase IV in tension
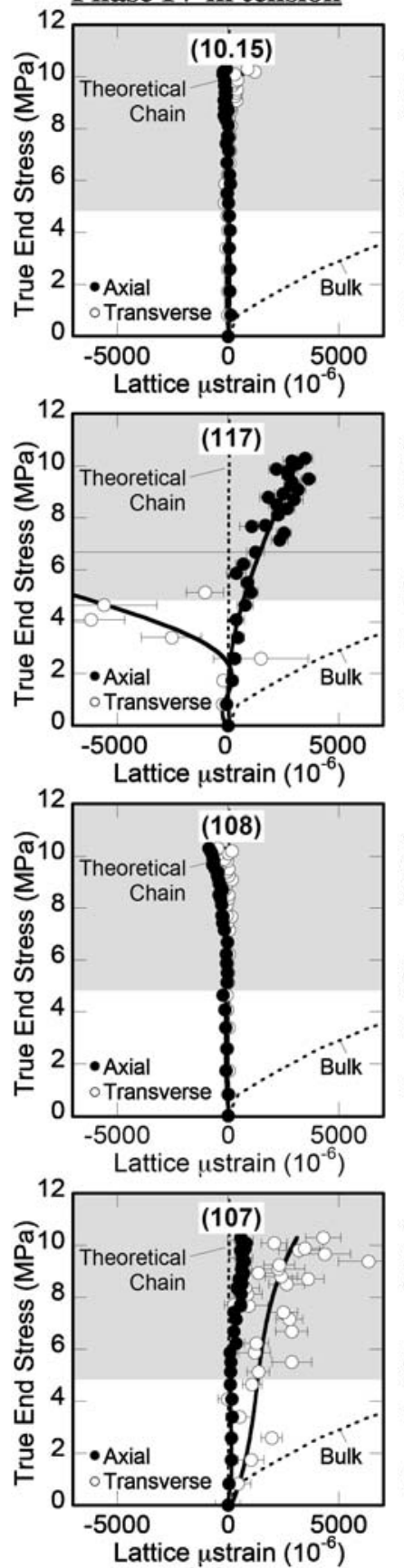

(a)
Phase IV in compression
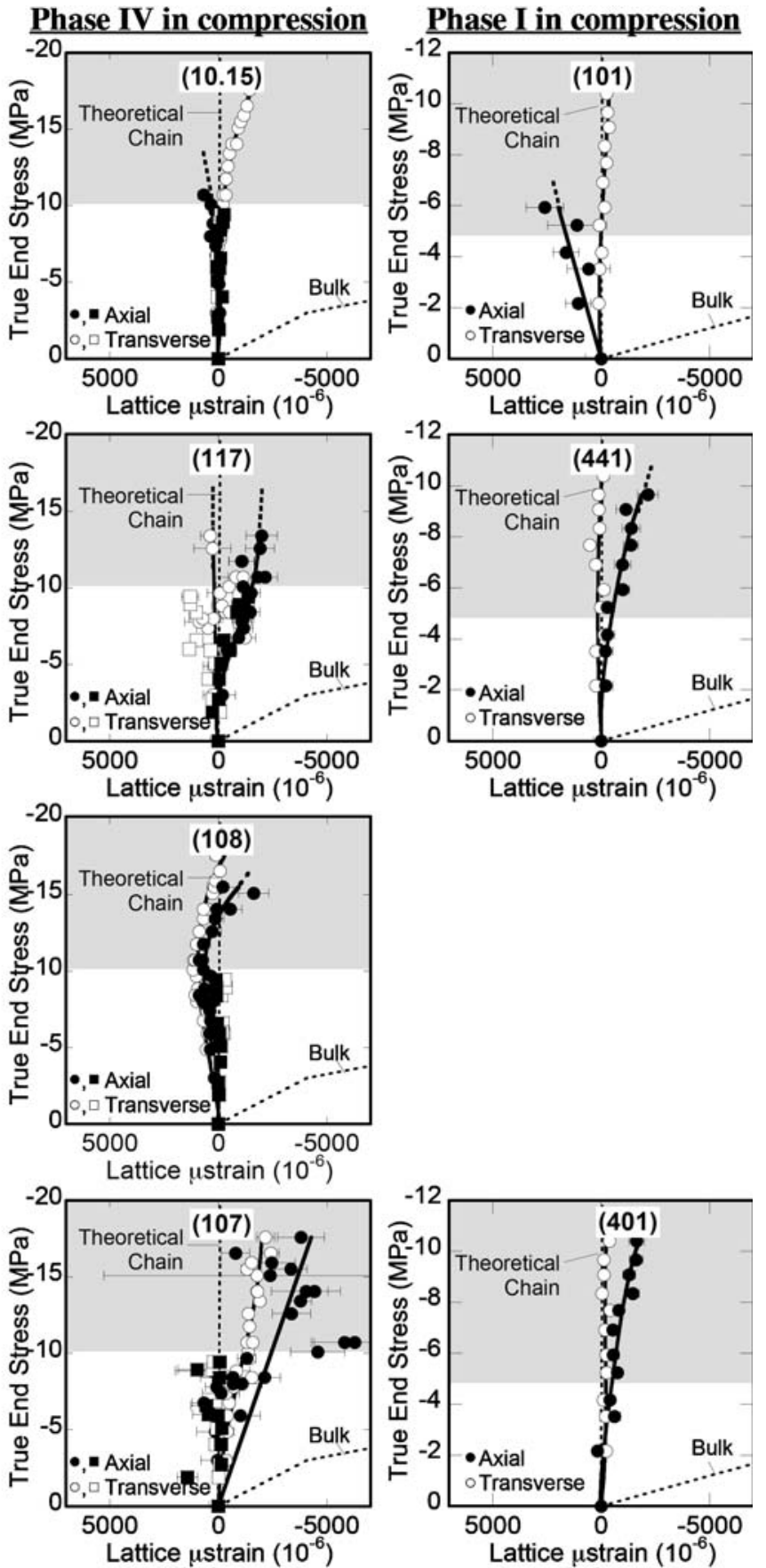

(b)



(c) in total intensity of the peaks consistent between tension and compression.

Diffraction elastic constants were determined based on the diffraction lattice strains and the far field true stress, given in Table 4. While neutron diffraction provides a new level of insight into the measurement of strains at the atomic scale, the authors are unaware of a comparable diagnostic for the in situ measurement of lattice specific atomic stresses. Therefore a constant-stress field assump- tion is adopted from composite theory. Elastic constants are calculated using the data taken prior to yield as

$E_{h k l}=\sigma^{f f} / \varepsilon_{h k l}$,

where $\sigma^{f f}$ is the far field applied true stress.

Deformation parallel to the prismatic plane normals exhibit a modulus $\sim 10 \times$ bulk $[1,2]$. Since crystalline PTFE is by definition thermodynamically constrained and has $13 \%$ less volume (i.e., $13 \%$ higher density) than amorphous 
Table 4 Elastic constants for prismatic and basal crystalline lattices

\begin{tabular}{llll}
\hline (hkl) & \multicolumn{3}{l}{ Elastic constants $(\mathrm{GPa})$} \\
\cline { 2 - 4 } & $\begin{array}{l}\text { Tension } \\
\text { RT }\end{array}$ & $\begin{array}{l}\text { Compression } \\
\text { RT }\end{array}$ & $\begin{array}{l}\text { Compression } \\
60^{\circ} \mathrm{C}\end{array}$ \\
\hline Bulk behavior & $0.5^{\mathrm{a}}$ & $0.6^{\mathrm{a}}$ & $0.2^{\mathrm{a}}$ \\
Theoretical PTFE chain & $220.5^{\mathrm{b}}$ & $220.5^{\mathrm{b}}$ & $\sim 220^{\mathrm{c}}$ \\
210 & 3.8 & 1.8 & 5.4 \\
300 & 4.8 & 5.1 & 3.0 \\
220 & 2.2 & 5.4 & 2.3 \\
310 & 4.1 & 5.8 & 3.1 \\
00.15 & na & $\sim 220$ & - \\
001 & - & - & $\sim 220$
\end{tabular}

${ }^{\text {a }}$ The bulk behavior is taken from [1,2] agrees well with the initial bulk response measured in SMARTS.

${ }^{\mathrm{b}}$ The theoretical elastic modulus is taken from [32] for $164.5^{\circ}$ screw angle.

${ }^{\mathrm{c}}$ The effect of screw angle from $164.5^{\circ}$ to $180^{\circ}$ is a difference of only $6 \mathrm{GPa}$ so the difference from phase IV with a perfect helix to phase I with random structure is assumed to be similar magnitude.

PTFE to accommodate chain movement, it is expected that the crystalline PTFE should be stiffer than the bulk polymer. For loading parallel to the prismatic plane normals deformation is dominated by inter-polymer chain compression that only requires driving against the relatively weak Van der Waals forces. Alternately, for loading parallel to the basal plane normals, deformation is dominated by intrapolymer chain compression that requires driving against the much stronger covalent forces. In this configuration the modulus is $\sim 1,000 \times$ bulk, corresponding with the theoretical value for a PTFE chain modulus of $220.5 \mathrm{GPa}$ [34] to within the measurement error bars.

\section{Pyramidal Plane Normal Behavior}

Deformation parallel to the pyramidal plane normalsphase IV (107), (117), (108), (10.15) and phase I (101) and (401) - is more complicated. While the magnitude of response in the loading and Poisson directions remains bounded by the compliant response of bulk PTFE and the stiff theoretical response of the PTFE polymer chain, the pyramidal planes exhibit an apparent negative Poisson effect that would require either large increases or decreases in volume. Both the axial and transverse strains in the (107), (117) and (401) planes in phase IV and I respectively follow the same sign as the applied load, while the (108), (10.15), and (101) planes exhibit both axial and transverse strains of the opposite sign as the applied load. At least at small stresses the strains are similar to the theoretical value for compression of the PTFE chain. While the lattice easily accommodates the loading component parallel to the prismatic plane normal, the strong covalent bonds of the polymer chain backbone resist deformation to accommodate the component of loading parallel to the basal plane normal. The fluorine atoms form a smooth cylinder around the central carbon atoms preventing chain side group entanglement that would otherwise impede shearing. Therefore, it is easier for the crystalline lattice to accommodate deformation by shearing along the prismatic planes, as modeled by Flack [37].

The proposed mechanisms incorporating shear enabling the apparent negative Poisson response of the lattice parameter, without requiring large volumetric changes, are illustrated in Fig. 10. Figure 10(a) illustrates an unloaded generic crystalline lattice with a measured pyramidal d-space. A representative volume of material is shown such that the vertical edge is parallel to the polymer chains. The application of axial and transverse loads on the representative volume are shown in Fig. 10(b), and separated into shear and normal components. It is worth noting that for all of the pyramidal planes considered in this work the angle between the plane and polymer chain (vertical) is significantly greater than $45^{\circ}$ (in volume space). The relevant two shear and two normal modes are shown in Fig. 10(c)-(f). The two simple shear conditions [Fig. 10(c) and (d)] assume no deformation between atoms on the polymer chain and constant normal distance between the polymer chains. From the observation that the lattice perpendicular to the prismatic plane normals is 2 orders of magnitude more compliant than the perpendicular to the basal plane normals, the normal conditions [Fig. 10(e and f)] assume negligible deformation between atoms on the polymer chain and focuses on changes in the normal distance between the polymer chains. It therefore follows that for axial loading, there is competition between a shear mode that reduces the $\mathrm{d}$-spacing and a normal mode that increases the d-spacing. There is a similar but reversed competition for transverse loading.

To isolate the dominant mode, the observed measurements (Fig. 9) are compared with the indeterminate predictions of changes in $\mathrm{d}$-spacing with competing shear and normal modes of deformation. The subset of measurements for (107), (117), (401), and (441) plane normals exhibit reduction in the $\mathrm{d}$-space for both the axial and transverse directions, indicating that these plane deform under a shear mode [Fig. 10(d)] for axial loading condition on under a normal mode [Fig. 10(f)] for transverse loading. Conversely, the subset of measurements for (108), (10.15), and (101) plane normals exhibit increases in the d-space for both the axial and transverse directions, indicating that these planes deform under a normal mode [Fig. 10(f)] for axial loading condition on under a shear mode [Fig. 10(e)] for transverse loading. In addition to these two subsets categorizing the observed strain response, they also have physical significance in terms of the crystal structure. While it was previously stated that for volume space (i.e., a 
Fig. 10 Proposed pyramidal deformation mechanisms incorporating shear. (a) Unloaded generic crystalline lattice with a measured pyramidal d-space. (b) Applied of axial and transverse loads on the representative volume with shear and normal components, and relevant shear (c and $\mathbf{d}$ ) and normal (e and $\mathbf{f}$ ) modes
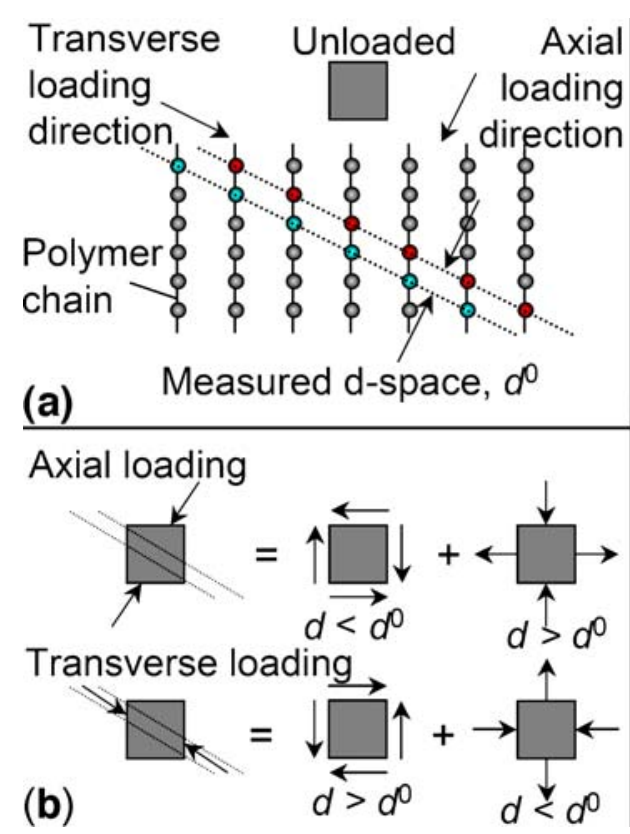

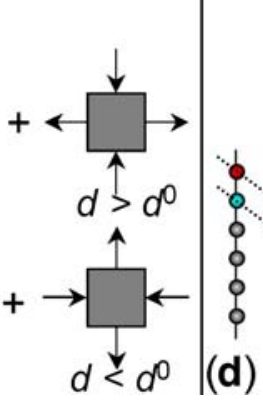

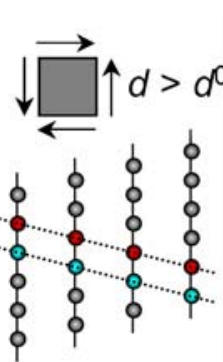

c)

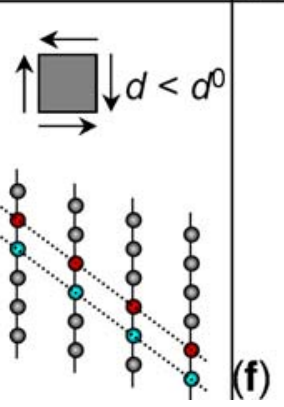

(e)

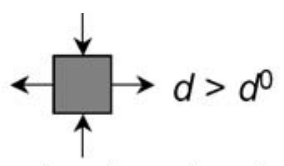



geometrical cube with units of $\mathrm{nm}$ ) the angle between the plane and polymer chain (vertical) is significantly greater than $45^{\circ}$ for all of the pyramidal planes considered in this work, the difference in the inter-atom spacing along a polymer chain versus the inter-atom spacing between polymer chains allows for the plane normals in the current work to tend more closely to either the prismatic or basal planes. In Fig. 10(a) the illustrated (101) plane represents the midpoint between the prismatic or basal planes (i.e. $45^{\circ}$ in atom space). All planes rotated clockwise from this midpoint tend towards more prismatic, which we denote as Type A pyramidal planes, and all plane rotated counterclockwise from tend towards more basal, which we denote as Type B pyramidal planes. The midpoint itself is considered to be Type B. For phase I PTFE this midpoint is the (101) plane and for phase IV PTFE is the nonphysical (10.7 1/2). Therefore, the (401) and (441) phase I planes and the (107) and (117) phase IV planes are all Type A, while the (101) phase I planes and the (108) and (10.15) phase IV planes are all Type B. It therefore follows that the shear deformation mode is dominant for Type A planes under axial loads and Type B planes under transverse loads. Both of these are cases where the angle between loading direction and the polymer chain axis is less than $45^{\circ}$ in atom space. The normal deformation mode is dominant for
Type B planes under axial loads and Type A planes under transverse loads, corresponding to the cases where the angle between loading direction and the polymer chain axis is greater than $45^{\circ}$ in atom space.

\section{Amorphous Behavior}

The structure of PTFE, as shown in Fig. 1(c), can be simplistically thought of as a particulate composite consisting of stiff crystalline PTFE particles surrounded by a much more compliant amorphous PTFE matrix. The neutron measurements have allowed us to directly determine information about the crystalline phase. Using a simple constant-stress rule-of-mixtures model it is possible to infer something about the amorphous phase. Using the DSC measurement of crystalline content is taken to be $38 \%$. Prior to applying of a far-field load equivalent diffraction patterns are observed in the axial and transverse detectors indicating a random texture in the PTFE, see [35]. Based on the random orientation of grains the crystalline phase is equally divided between the ten unique crystallographic orientations listed in Table 2 (i.e., the reflections of (100), (200), and (300) and (110) and (220) are each only represented once to address the multiplicity of different orientations), the amorphous elastic modulus is given by

$E_{\text {amor }}=0.62\left[E_{\text {bulk }}^{-1}-0.038\left[E_{107}^{-1}+E_{108}^{-1}+E_{117}^{-1}+E_{210}^{-1}+E_{118}^{-1}+E_{300}^{-1}+E_{220}^{-1}+E_{310}^{-1}+E_{00.15}^{-1}+E_{10.15}^{-1}\right]\right]^{-1}$

The bulk, prismatic normal, and basal normal elastic constants are taken from Table 4. Although elastic moduli are not reported for the pyramidal normal response due their shear behavior, their axial response tends to approach 
the theoretical response. Moreover, the solution to equation 5 is sufficiently insensitive that $E_{107}, E_{108}, E_{117}, E_{118}$, and $E_{10.15}$ can be varied between the maximum and minimum elastic constants of the prismatic normal and basal normal responses without notably changing the prediction for the amorphous modulus (the error introduced is between 1 and $5 \%$ ). At room temperature the elastic modulus of the amorphous PTFE is 0.32 and $0.38 \mathrm{GPa}$ in tension and compression respectively. At $60^{\circ} \mathrm{C}$ under compression the elastic modulus of the amorphous PTFE is $0.13 \mathrm{GPa}$. This shows the elastic modulus to be less than modulus of the bulk or crystalline domains, both of which are expected. Moreover, it shows that the modulus of the more compliant amorphous phase is of the same order as the bulk, dominating the bulk response despite being $\sim 1000 \times$ more compliant than the stiffest crystalline orientation. This simple analysis also supports the observation that the modulus of PTFE increases with increasing crystallinity [2].

\section{Conclusions}

Strain measurements by neutron diffraction have been employed as an in situ strain measurement technique to obtain insight into the deformation modes of crystalline domains in a deformed semi-crystalline polymer. The crystalline lattice displacements in polytetrafluoroethylene (PTFE) for crystalline phase IV (at room temperature) in tension and compression and for crystalline phase I (at $60^{\circ}$ C) in compression have been measured. The chemical structure of PTFE, $\left(\mathrm{C}_{2} \mathrm{~F}_{4}\right)_{\mathrm{n}}$, makes it ideally suited for investigation by neutron methods as it is free of the hydrogen that results in limited penetration depths and poor diffraction acquisition in most polymers. Deformation parallel to the prismatic plane normals is shown to occur by inter-polymer chain compression with a moduli $\sim 10 \times$ bulk, while deformation parallel to the basal plane normal occurs by intra-polymer chain compression with a moduli $\sim 1,000 \times$ bulk, corresponding with theoretical values for a PTFE chain modulus. The amorphous modulus is calculated to be $\sim 65 \%$ that of the bulk by a simple constant-strain rule-ofmixtures model.

Acknowledgments This research was supported under the auspices of the US Department of Energy operated by the University of California and the Joint DoD/DOE Munitions Program. This work has benefited from the use of the Lujan Neutron Scattering Center at LANSCE, which is funded by the Department of Energy's Office of Basic Energy Sciences. Los Alamos National Laboratory is operated by Los Alamos National Security LLC under DOE Contract DEAC52-06NA25396. The authors wish to thank T. Sisneros for his assistance with experimental setup and J. Bingert, M. Bourke, and S. Vogel for many helpful discussions.

\section{References}

1. Rae PJ, Brown EN (2005a) The properties of poly(tetrafluoroethylene) (PTFE) in tension. Polymer 46(19):8128-8140.

2. Rae PJ, Dattelbaum DM (2004) The properties of poly(tetrafluoroethylene) (PTFE) in compression. Polymer 45(22):76157625.

3. Brown EN, Willms RB, Gray GT III, Rae PJ, Cady CM, Vecchio KS, Flowers J, Martinez MY (2007) Influence of molecular conformation on the constitutive response of polyethylene: a comparison of HDPE, UHMWPE, and PEX. Exp Mech 47 (3):381-393.

4. Vaidyanathan R, Bourke MAM, Dunand DC (1999) Phase fraction, texture and strain evolution in superelastic niti and nititic composites investigated by neutron diffraction. Acta Mater 47 (12):3353-3366.

5. Clausen B, Lorentzen T, Bourke MAM, Daymond MR (1999) Lattice strain evolution during uniaxial loading of stainless steel. Mater Sci Eng A 259(1):17-24.

6. Brown DW, Bourke MAM, Dunn PS, Field RD, Stout MG, Thoma DJ (2001) Uniaxial tensile deformation of uranium $6 \mathrm{wt}$ pct niobium: a neutron diffraction study of deformation twinning. Met Maters Trans A 32(9):2219-2228.

7. Agnew SR, Brown DW, Tome CN (2006) Validating a polycrystal model for the elastoplastic response of magnesium alloy AZ31 using in situ neutron diffraction. Acta Mater 54(18):4841-4852.

8. Brown DW, Bourke MAM, Field RD, Hults WL, Teter DF, Thoma DJ, Vogel SC (2006) Neutron diffraction study of the deformation mechanisms of the uranium- 7 wt.\% niobium shape memory alloy. Mater Sci Eng A 421(1-2):15-21.

9. Clausen B, Bourke MAM, Brown DW, Üstündag E (2006) Load sharing in tungsten fiber reinforced kanthal composites. Mater Sci Eng A 421(1-2):9-14.

10. Poulsen HF, Wert JA, Neuefeind J, Honkimaki V, Daymond M (2005) Measuring strain distributions in amorphous materials. Nat Mater 4(1):33-36.

11. Bunn CW, Howells ER (1954) Structure s of molecules and crystals of fluorocarbons. Nature 174(4429):549-551.

12. Sperati CA (1961) Fluorine-containing polymers, II: polytetrafluoroethylene. Fortschr Hochpolym-Forsch Bd 2:465-495.

13. Bunn CW, Cobbold AJ, Palmer RP (1958) The fine structure of polytetrafluoroethylene. J Polym Sci 28(117):365-370.

14. Clark ES (1999) Molecular conformations of polytetrafluoroethylene: forms II and IV. Polymer 40(16):4659-4665.

15. Weeks JJ, Sanchez IC, Eby RK (1980) Order-disorder transitions in polytetrafluoroethylene. Polymer 21(3):325-331.

16. Cox JM, Wright BA, Wright WW (1964) Thermal degradation of fluorine-containing polymers - 1, 2. J Appl Polym Sci 8(6):2935-2961.

17. Brown EN, Trujillo CP, Gray GT, Rae PJ, Bourne N (2007) Softrecovery of polytetrafluoroethylene shocked through the crystalline phase II-III transition. J Appl Phys 101(2):024916.

18. Hosemann R (1962) Crystallinity in high polymers, especially fibres. Polymer 3(3):349-392.

19. Lehnert RJ, Hendra PJ, Everall N, Clayden NJ (1997) Comparative quantitative study on the crystallinity of poly(tetrafluoroethylene) including Raman, infra-red and F-19 nuclear magnetic resonance spectroscopy. Polymer 38(7):1521-1535.

20. Brown EN, Dattelbaum DM (2005) The role of crystalline phase on fracture and microstructure evolution of polytetrafluoroethylene (PTFE). Polymer 46(9):3056-3068.

21. Rae PJ, Brown EN, Clements BE, Dattelbaum DM (2005b) Pressure-induced phase change in poly(tetrafluoroethylene) at modest impact velocities. J Appl Phys 98(6):63521-63521-8. 
Exp Mech (2008) 48:119-131

131

22. Brown EN, Rae PJ, Orler EB, Gray GT, Dattelbaum DM (2006a) The effect of crystallinity on the fracture of polytetrafluoroethylene (PTFE). Mater Sci Eng C 26(8):1338-1343.

23. Brown EN, Rae PJ, Gray GT (2006c) The influence of temperature and strain rate on the tensile and compressive constitutive response of four. J Phys IV 134:935-940.

24. Brown EN, Rae PJ, Diu C (2007) Mixed-Mode I/II Fracture of Polytetrafluoroethylene. Mater Sci Eng A 2006, in press DOI 10.1016/j.msea.2006.09.125.

25. Burke MAM, Dunand DC, Ustundag E (2002) SMARTS-a spectrometer for strain measurement in engineering materials. Appl Phys A Mater Sci Process 74(suppl. II):s1707-s1709.

26. SMARTS, Available from: < http://lansce.lanl.gov/lujan/ER1ER2/ SMARTs/index.html>.

27. Yon Dreele RB, Jorgensen JD, Windsor CG (1982) Rietveld refinement with spallation neutron powder diffraction data. J App Crystallogr 15:581-589.

28. Sisneros TA (2006) J Neutron Res in press.

29. Kimmig M, Strobl G, Stühn B (1994) Chain reorientation in poly(tetrafluoroethylene) by mobile twin-helix reversal defects. Macromolecules 27(9):2481-2495.

30. Weeks JJ, Clark ES, Eby RK (1981) Crystal structure of the low temperature phase (II) of polytetrafluoroethylene. Polymer 22 (11):1480-1486.
31. Clark ES, Muss LT (1962) Partial disordering and crystal transitions in polytetrafluoroethylene. Z Kristallogr Kristallgeom Kristallphy 117:119-127.

32. Clark ES (1967) Molecular motion in polytetrafluoroethylene at cryogenic temperatures. J Macromol Sci Phys B1(4):795800 .

33. Bouznik VM, Kirik SD, Solovyov LA, Tsvetnikov AK (2004) A crystal structure of ultra-dispersed from of polytetrafluoroethylene based on X-ray powder diffraction data. Powder Diffr 19(3):219224.

34. Barth F, Boyar F, Peters A, Van Alsenoy C, Van Loren V (2000) Density-functional calculations of the elastic properties of some polymer chains. Phys Rev B 62(15):1014210150.

35. Brown EN, Dattelbaum DM, Brown DW, Rae PJ, Clausen B (2007) A new strain path to inducing phase transitions in semicrystalline polymers. Polymer 48(9):2531-2536.

36. Brown EN, Rae PJ, Orle EB, Thissell WR, Dattelbaum DM (2004) Fracture and damage evolution of fluorinate polymers. In: Proulx T (ed) Proceedings of the 2004 SEM $\mathrm{X}$ International Congress and Exposition on Experimental and Applied Mechanics.

37. Flack HD (1974) Sliding in polytetrafluoroethylene crystals. J Polys Sci 12(1):81-87.

SEM 